\title{
Review
}

\section{Digital Cytology: Current State of the Art and Prospects for the Future}

\author{
David C. Wilbur \\ Department of Pathology, Massachusetts General Hospital, Harvard Medical School, Boston, Mass., USA
}

\section{Key Words}

Cytology $\cdot$ Telecytology $\cdot$ Telepathology $\cdot$ Digital imaging $\cdot$ Whole slide $\cdot$ Images

\begin{abstract}
The growth of digital methods in pathology is accelerating. Digital images can be used for a variety of applications in cytology, including rapid interpretations, primary diagnosis and second opinions, continuing education and proficiency testing. All of these functions can be performed using small static digital images, real-time dynamic digital microscopy, or whole-slide images. This review will discuss the general principles of digital pathology, its methods and applications to cytologic specimens. As cytologic specimens have unique features compared to histopathology specimens, the key differences will be discussed. Technical and administrative issues in digital pathology applications and the outlook for the future of the field will be presented.
\end{abstract}

Copyright ๑ 2011 S. Karger AG, Basel

\section{Introduction}

The pace of development in digital pathology is accelerating. With recent increases in computer processing power and storage capability, internet and data transfer speed, and new software development, the use of digital images for a wide variety of functions is being realized. This functionality is at multiple levels, ranging from relatively simple tasks such as image/case archiving, to the mid-level tasks of interactive continuing education, teleconsultation and proficiency testing, and culminating at the higher-level tasks of image data-mining and the development of artificial intelligence tools.

The practice of cytology has been, and will continue to be, altered by the emergence of digital methodologies. However, compared to digital pathology applications in surgical pathology, the cytologic specimen has unique features that require special functionality and approaches. Histopathology specimens are highly adaptable to simple, single-plane digital scanning because of the very nature of their preparation - that being as relatively flat, single plane sections. Thus, single-plane high-resolution digital scans recapitulate the original specimen very closely. Cytology specimens, on the other hand, are intrinsically three-dimensional, requiring special manipulations in order to produce digital renditions that truly recapitulate the original specimen (fig. 1). Therefore, although digital cytology has similar applicability to all the functionality noted above, in practice its adaptability is more challenging.

This review will provide a snapshot of the current state of general digital pathology applications available, will

\section{KARGER \\ Fax +4161306 1234 \\ E-Mail karger@karger.ch}

www.karger.com
(C) 2011 S. Karger AG, Basel

$0001-5547 / 11 / 0553-0227 \$ 38.00 / 0$

Accessible online at:

www.karger.com/acy
Correspondence to: Prof. David C. Wilbur

Department of Pathology, Massachusetts General Hospital, Harvard Medical School 55 Fruit Street, Warren 120

Boston, MA 02114 (USA)

Tel. +1 617726 7943, E-Mail dwilbur@partners.org 


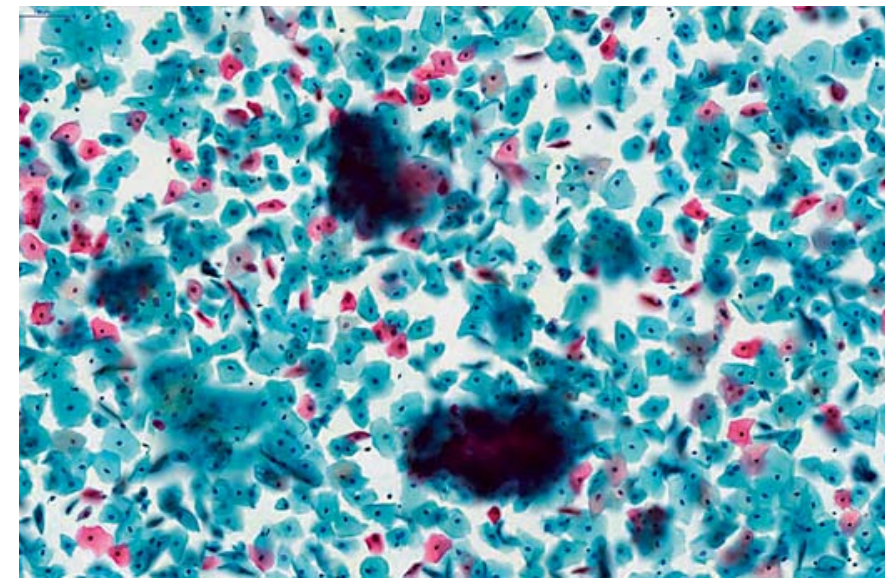

Fig. 1. Example of a whole-slide image showing the 3-dimensional nature of a cytology specimen. Note that in this image most cells are in focus while others, particularly in the 3 cell groupings, are out of focus because the specimen was scanned in a single plane selected by the scanning device.

present the specific challenges faced by adaptation of these applications to cytologic practice, detail data accumulated to date regarding cytologic applications in digital pathology, and conclude with a look at the future with regard to specific digital-pathology issues and solutions.

\section{The Basics of Digital Pathology}

Digital pathology represents a fundamental change in the format of the images that practitioners routinely view. Instead of viewing analog images through an opticsbased device (i.e. a microscope), digital pathology is most basically defined as viewing digital renditions of those same images through a digital monitor. In order to do so, analog images must first be captured. This requires a device, such as a camera or scanner, that renders the analog image into an array of 'dots' referred to as pixels, each of which has a specific color representative of the color that was present in the analog image at that point. When the entire array of pixels is viewed on an output device, it forms a complete rendition of the original image. The digital images that derive from home-use digital cameras are a simple example of this functionality. The resolution of the image is determined by the number and size of the pixels in the array, and as any photographer will know, the more pixels in the image, the larger and more finely resolved the final image can be without distortion (socalled 'pixelation') of the image (fig. 2). Resolution comes with costs, including higher memory requirements to store the data, and the need for higher bandwidth capacity to transfer those images to remote sites over networks, such as the Internet. So as cameras have become more powerful (meaning that they can capture images containing greater pixel arrays), the ability to practically digitize pathology specimens at high enough resolutions is now a reality. In addition, with the development of highresolution scanners, which are essentially cameras that digitize images across large arrays and combine those images (so-called 'stitching') into yet larger arrays, it is now possible to fabricate final image files that recapitulate entire pathology specimens (so-called 'whole-slide images' or 'virtual slides'). In addition, digital cameras can also be used to 'stream' images over networks without the need to create files or to use permanent computer memory for storage. Thus, real-time review of digitized specimens can take place using digital technology with bandwidth requirements only for transferring the image being viewed at any particular moment.

\section{Methods of Digital Pathology Image Transfer}

As noted above, there are 3 basic digital pathology image transfer methods currently in common use. Transfer of static images, real-time (or streaming) image transfer, or the transfer of whole-slide images.

Static-image transfer has the advantage of ease of use and relatively low cost. This process requires only a camera, computer and network connection to operate. Image size (as compared to whole-slide images) is small and bandwidth requirements are low. However, static images show only a limited portion of the entire specimen. Therefore, remote observers will only see what the originator has imaged. This situation may be perfectly acceptable for education and testing purposes, but this process creates a bias which may be detrimental in the particular circumstance of telediagnosis/consultation in which the specimen is an unknown for which a diagnostic opinion is being sought. In 'simple' cases this may not be a significant issue, but in difficult or true 'consultation grade' cases, this bias could potentially skew the opinion rendered. The lack of any ability to focus, change magnifications, or otherwise manipulate the image, can also be detriments to optimal interpretation with static-image transfer. One study used video image capture as a method of solving the focusing problem with static imagery. The authors report that using a specialized digital camera capable of capturing short video clips of manual focusing (a 
Fig. 2. Example of a pixelated image. Note the lower-magnification image on the left top $(20 \times)$ which is within the resolution window of the original scan's magnification which was performed with a $20 \times$ objective. In the lower left, the image is magnified to $40 \times$ which represents 'pseudomagnification'. This is still a useable image for most applications but may not be optimal for searching, especially for small cells or objects. The image on the right is the same cell as noted by the circles on the left, digitally magnified to $300 \times$. This magnification beyond resolution shows degradation (pixelation), leading to a 'fuzzy' image.

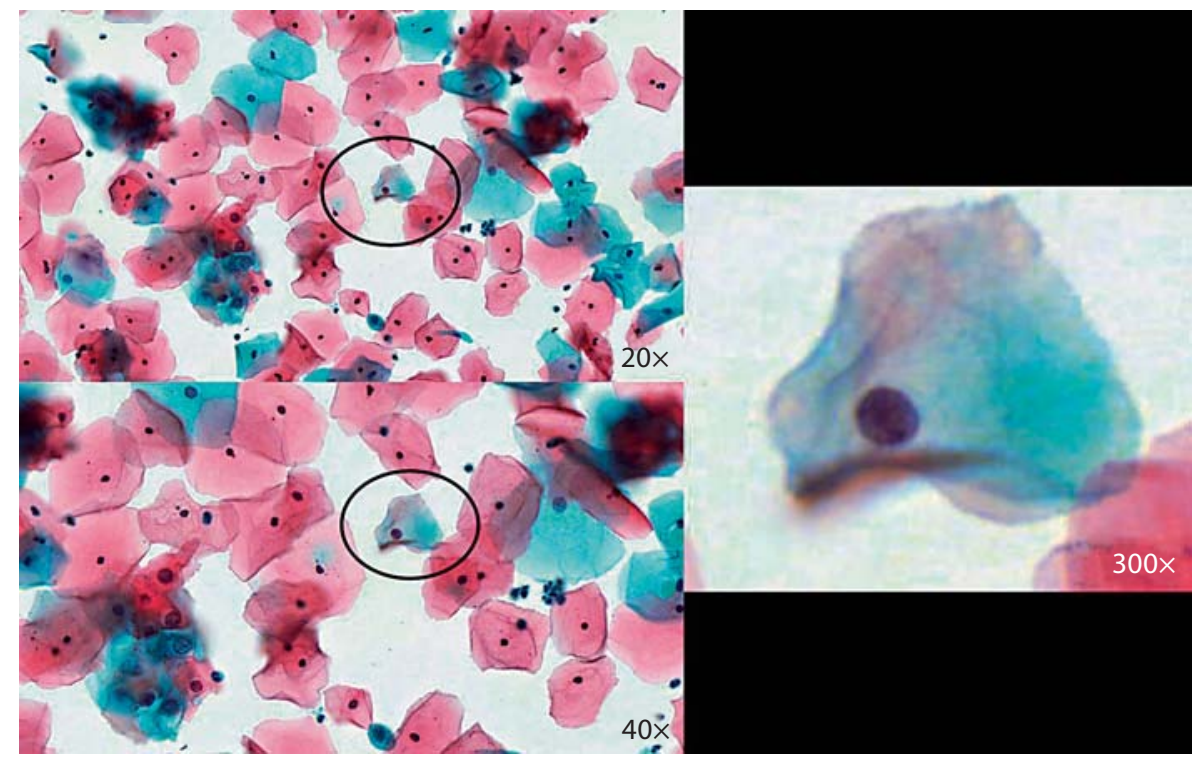

' $\mathrm{z}$-axis video') improved diagnostic ability compared to nonfocused still images [1]. When the review has been completed, the images can be permanently archived.

Real-time image transmission or streaming of a digital image solves some of the problems of static-image transfer, but at a cost. In this process, a microscope at the originating site actually views the specimen, and a continuously updated digital image is streamed over the network to the remote site. The microscope can be either locally or remotely controlled (the latter via a robotic interface), with commands sent to move the slide, and change the focus or magnification. Therefore the entire slide can be visualized in a manner entirely analogous to primary microscopy, thus eliminating the bias of static imaging. However, this process can be exceedingly cumbersome, as the manipulation of the remote microscope can be tedious and slow. Real-time systems work best for histopathology applications because much of the work is done at lower magnifications, allowing greater areas of the slide to be viewed in a smaller amount of time. Real-time imaging is least useful for applications requiring high magnification searching or screening, hence, its use for cytologic material is most limited. Real-time high-resolution image transfer requires high bandwidth and can easily overload network capacity. Lower-resolution systems require less bandwidth, but suffer from lack of fine detail. When the real-time review is completed, no stored image or record of the review exists, unless pictures are recorded during the session. Real-time remotely controlled robotic devices are expensive; however, locally controlled real-time image systems are inexpensive and can be efficiently deployed.

Whole-slide imaging maintains the relative simplicity of static-image transfer (in reality, a whole-slide image is merely a very large static image), but eliminates its bias because the entire specimen is available for review. This process creates a very large file size for each image (wholeslide images can be many hundreds of megabytes of data each), and hence the memory required to store such images is great. At present, the whole-slide image-scanning process is lengthy. A single plane scan can take up to 5 min depending on the area of the specimen, and with the higher-resolution scanning that will optimize the high magnification ( $40 \times$ objective scanning) image for cytology specimens, scanning can take $30-40 \mathrm{~min}$. Faster scanner mechanics or multiple-array objectives will undoubtedly mitigate this scanning time process in the future [2]. As with static imaging, whole-slide images share the lack of intrinsic focusing capability. For cytologic material, which is often displayed in a mutiplanar fashion, this can be a detriment, although there are methods to mitigate the 3-dimensional aspects of cytologic whole-slide images. Multiplane scanning of a specimen (so-called 'z-stacking') allows for capture of many focal planes, with the ability of the viewer to 'focus' up and down through the planes to find optimally focused cells in each area of the specimen. $z$-Stacking requires the storage of file sizes that are multiples of the already large single-plane whole-slide images. Systems currently in use to transfer z-stacks over networks can be very slow and can 


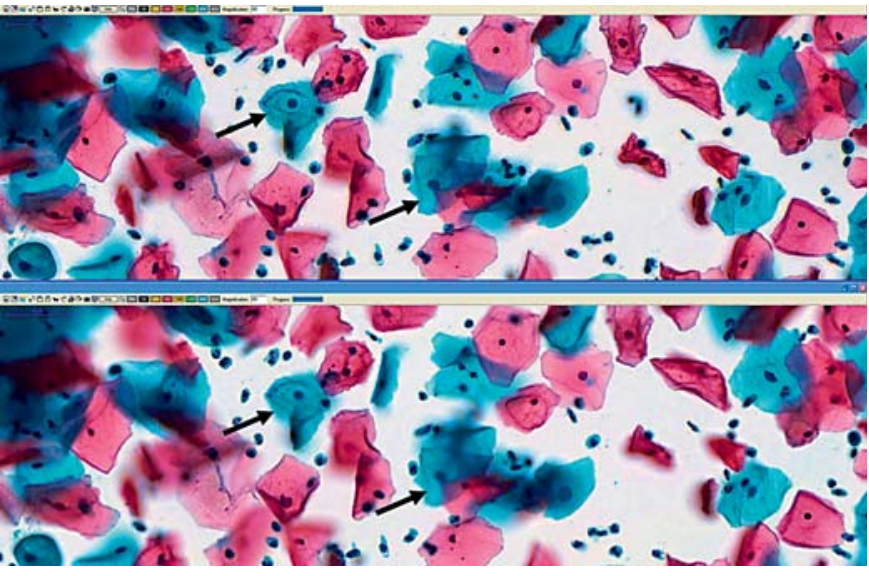

Fig. 3. Example of a multiplane scan whole-slide image in which each of the planes has been condensed (or integrated) into a final single plane for viewing. This process identifies the best focused plane in each area and incorporates it into the final image. The upper figure is a condensed final image derived from a 9-plane scan whereas the bottom image is a single-plane scan. Note at the arrows significant improvement in focus of the cells in the 9-plane scan. Integration is not a final answer because it will not resolve groups of cells or cells overlapping one another, but it improves the focus over single-plane scanning as was illustrated in figure 1.

therefore lead to user fatigue and frustration [3]. Going forward, these downside issues of z-stacking may be viewed as 'engineering' problems that will be solved with faster scanners, computers and networks; and with everincreasing storage capacities. Alternative solutions to zstacking for the 3-dimensionality of cytologic specimens have been achieved using scanners that allow for multiplane scanning with integration of the best focused image at each tile into the final single-plane file. Scanning time is not improved over z-stacking as each plane still needs to be scanned individually, but the final integrated file uses the memory and transfer requirements of a singleplane scan. Focus of the whole-slide image has been shown to be significantly improved using this method (fig. 3). Interestingly, a study aimed at defining differences in performance of observers using 2-dimensional versus 3 -dimensional cytology images showed no difference in diagnostic accuracy; however, it did highlight a higher level of 'uncertainty and frustration' of the observers who were unable to resolve out-of-focus cells on the 2-dimensional whole-slide images [4]. At present, a major limiting factor in the use of whole-slide images is the high cost of the scanning devices. However, bandwidth requirements for whole-slide image transfer are not onerous because modern transfer techniques do not require transfer of the entire image file. The image file resides on the remote server and only the pieces of the image being queried at any point are transferred. Newer programs also increase the speed of viewing by also transferring the surrounding areas in anticipation of the observer's next move. An example of a typical viewing station for a whole-slide image is illustrated in figure 4 . See table 1 for a summary of the features of the 3 methods of digital image transfer described above.

\section{Applications of Digital Pathology}

Using digitized images, be they individual fields of a larger pathology specimen or whole-slide images, a variety of practical applications are in common use or are being investigated. Image archiving is a basic use with which all will be familiar. Virtually all photographs of pathology material are currently taken in the digital format. Use of film-based media for image capture and storage is all but gone. Images used in lectures, textbooks, teaching sets, and for illustrations on patient pathology reports are all captured using the digital format and can therefore be stored on digital storage media. Compared to film-based images, digital images have significant advantages - they can be stored in databases for simple cataloguing and retrieval, can be duplicated with a click of the 'save as' function on a computer, and can be transmitted to any other computer in the world that is linked by a network.

This ability to transfer digital images brings us to the functionality which has been referred to as 'telepathology' - or the ability to review pathologic specimens at a distance using image transfer over networks. Telepathology applications are many and include the following: (1) telediagnosis/teleconsultation - the rendering of an opinion on digitally transferred images or entire digital specimens (either primary diagnosis or second opinions); (2) continuing education - the use of digital images/specimens to teach; (3) proficiency testing - the use of standardized digital images/specimens to test individuals' ability to render correct interpretations. Several excellent reviews of general telepathology applications are available for the interested reader [5-7]. The remainder of this review will focus specifically on these applications in the field of cytology. In 1997, a 'Task Force on Digital Imagery/Telecytology, Diagnostic Cytology Toward the 21st Century: An International Expert Conference and Tutorial' was convened in Hawaii, which highlighted many of the issues relevant to the field going forward. The detailed review of that session was published in 1998 [8]. 
Fig. 4. Example of a whole-slide image review station screen. The primary viewing area is on the right side of the image. 'Thumbnail' images for localization are shown on the left. Magnification is controlled by the buttons on the top of the screen (arrow) with the actual magnification shown in the box (double arrow), which is $10 \times$ in this example. In this viewer, a high-magnification 'hover' window is present in the upper right corner of the viewer (triple arrow), allowing the observer a quick view of the area covered by the mouse cursor.

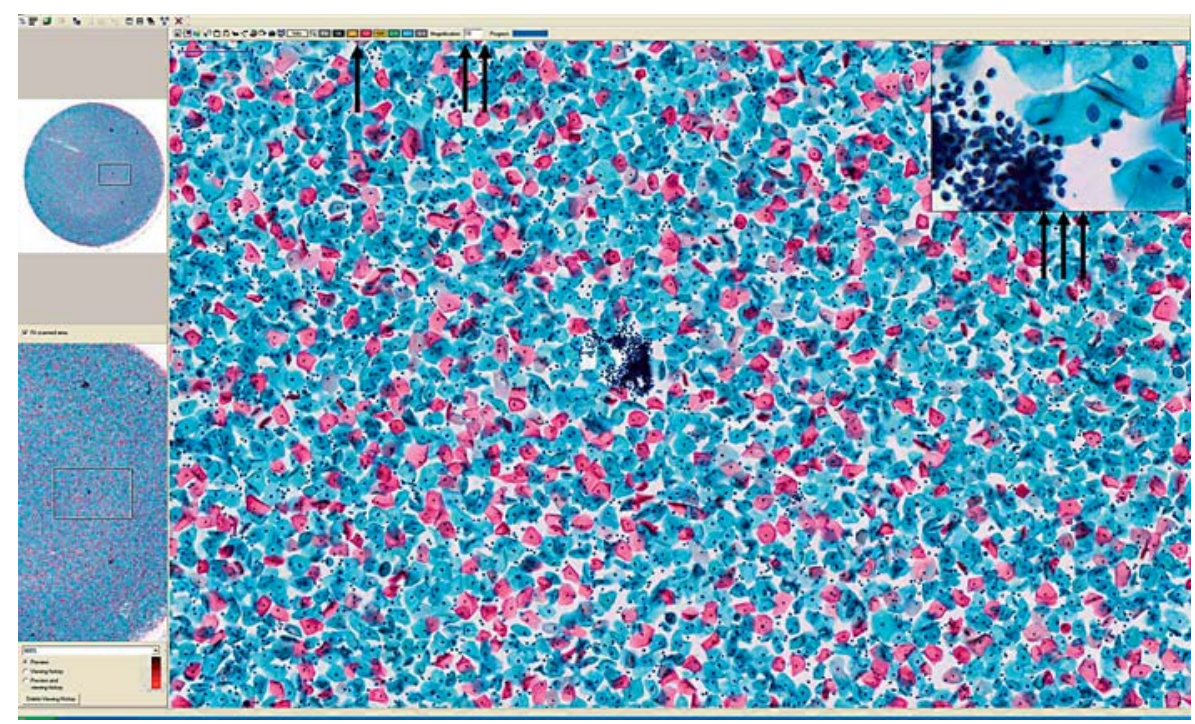

\section{Telediagnosis/Teleconsultation}

The need for remote interpretation of cytologic specimens has 3 basic applications; rapid adequacy assessment, primary specimen diagnosis, and second-opinion consultation. Each of these applications exploits the need to have cytology expertise in places where it does not ordinarily exist. This distance can be across hospitals (allowing busy pathologists to avoid the time of travel necessary within institutions), from hospital to hospital within networks, from healthcare facilities to home after hours, or from anywhere in the world having a fast Internet connection. Studies have shown the utility of using telepathology in the real-time mode for rapid interpretations [9-14]. In one study using real-time dynamic telecytology to perform rapid assessments of pancreatic fine-needle aspiration biopsies, Kim et al. [10] reported no significant disagreement between routine on-site and telecytology interpretations. They did note that the $\kappa$ agreement values were lower for telecytology, but that the results were not significant. Kerr et al. [12] reported $97 \%$ concordance and $99 \%$ accuracy using a locally controlled real-time system for rapid assessment. Alsharif et al. [13] reported only a $1.8 \%$ discrepancy rate using a real-time telecytology system, compared to a $3.1 \%$ discrepancy rate for routine glass-slide rapid evaluation, when each was compared to the final diagnosis. Their study was the largest to date, with over 400 cases analyzed. Heimann et al. [14] reported that the use of real-time telecytology for a variety of rapid fine-needle aspiration assessments compared favorably to on-site rapid interpretations. This report
Table 1. Comparative features of static-image, real-time analysis and whole-slide image methods

\begin{tabular}{|c|c|c|c|}
\hline Feature & Static & Real time & Whole slide \\
\hline Speed of acquisition & quick & medium & slow \\
\hline Speed of viewing & quick & slow & medium \\
\hline Focus capability & none & focusable & may be either \\
\hline Amount of specimen & portion & whole & whole \\
\hline Selection bias & yes & no bias & no bias \\
\hline Magnification & high & high & $\begin{array}{l}\text { dependent on } \\
\text { acquisition } \\
\text { magnification }\end{array}$ \\
\hline Permanent record & yes & no & yes \\
\hline Annotation & yes & $\begin{array}{l}\text { possible but } \\
\text { not routine }\end{array}$ & yes \\
\hline
\end{tabular}

noted that specimens prepared with Diff-Quik stains and those derived from endoscopic bronchial ultrasoundguided biopsies were more difficult to interpret. However, these issues may have been associated with both operator preference and experience. Overall, they reported that 95 and $97 \%$ of all specimens were concordant with the final and on-site rapid interpretations, respectively. These initial studies have also brought to light issues of work flow important to telecytology and the rapid-interpretation process [15]. With the rise in the use of fineneedle aspiration biopsy, more biopsies are being performed in more places, and for optimization will require more attention from the cytologist. The use of telecytology is seen as a less disruptive method of delivering that 
service because a small number of trained cytology resources (cytotechnologists and cytopathologists) will be able to cover many sites of biopsy performance from a single 'connected' office. As the technology for static or real-time telecytology becomes less expensive, this method should thrive to optimize the biopsies that are performed, ultimately leading to better and more cost-effective patient outcomes. The use of whole-slide imaging for rapid on-site interpretations is limited by the cost of scanning devices and by the technical issue of liquid slidemounting media and the need to be extremely careful to insure that buildup of media inside the device does not interfere with the day-to-day scanning process. These machines require meticulous cleaning to operate properly. For cytology rapid assessment, this problem is analogous to the same issue raised with frozen-section wholeslide imaging applications [7].

In the literature to date, static-image telecytology has been most commonly used for both primary diagnosis and second opinions. In most studies, static-image telecytology was at or near the accuracy of glass slide evaluation. The first reported telecytology study was performed in 1968 and consisted of using black and white videomicroscopy to transmit images of blood films from Logan International Airport in Boston to Massachusetts General Hospital as part of a larger telemedicine demonstration project [16].

Cervical cytology, being the highest-volume procedure in the cytology laboratory, has a history of evaluations with telecytology applications. Raab et al. [17], using static imagery for cervical cytology specimens, reported on the reproducibility of interpretation between video screen-based images and use of the microscope. The group found that use of the monitor-based diagnosis was about $10 \%$ less accurate than was microscopy, with most of the errors being false negatives of missed dysplasias. The authors did comment, however, that performance varied between individuals, raising the issue that training (and reorientation of skills) may be an important component of implementation of telepathology methods. In another study reviewing the ability to make accurate cervical cytology diagnoses on selected video images, Ziol et al. [18] noted that the process was generally accurate but suffered from the issues noted above in the general discussion for static-image telecytology - namely the inability to focus and lack of resolution, particularly for small cells. Alli et al. [19] reported that using selected static images in cervical cytology specimens, digital images generated lower $\kappa$ correlation statistic values than did glass slide interpretations, but also reported that no 'significant' 2-step diagnostic disagreements were noted on the digital images. They concluded that the method was therefore safe ['does not appear to pose a major risk of introducing large (2-step) diagnostic disagreements'] and 'holds much promise'. In 2001, the Armed Forces Institute of Pathology published their results for telecytology by static-image interpretation. This report was more relevant than the prior studies because it represented the compiled data of actual consultations received between 1995 and 1999, and not a contrived study. In this report, which included 15 cervical cytology and a variety of other nongynecologic specimens, the authors report no major discrepancies when compared to the actual consultations subsequently rendered on the glass slides. Minor discrepancies, such as upgrading of a telecytology interpretation of atypical squamous cells, cannot exclude a high-grade lesion, to high-grade squamous intraepithelial lesion were the only issues noted [20]. These types of discrepancies are similar to those reported in the abovementioned studies by Raab et al. and Ziol et al. Lee at al. [21], using cervical cytology, showed that static images transmitted by e-mail could be accurately and reproducibly diagnosed, and also noted that time to arrive at the diagnosis was less for static imagery (this result might be expected if preselected fields were obtained, as digital images mitigated the need to search for abnormality).

In parallel to the development of telecytology, the field of cervical cytology automated screening has been steadily progressing. At present, automated Pap test readers are available that, via artificial intelligence, identify slides at high risk of containing abnormal cells [22], but that can also identify which individual fields of view on slides are most likely to contain abnormal cells $[23,24]$. With the ability to identify high-risk fields of view, image capture of such fields with transmission of the static images to remote sites is a potential mode for 'automated telecytology.' This principle was first tested in a report using bundled low-resolution images of high-risk fields of view captured from an automated screening device [25]. In this report, the feasibility of such a system was proven, with high sensitivity and specificity for appropriate case triage by this method. Further studies using larger numbers of cases, with a system developed to automatically transmit images directly from the automated instrument to remote computer review stations, showed effective triage of specimens with a sensitivity of detection as high as has been found for primary manual screening by glass slide microscopy. In the static-image system, observers tended to overcall cases, leading to diminished specificity, but in a remote-triage application, use of this system would 
eliminate significant workload at the remote site by excluding cases deemed to represent the lowest risk of having abnormality with little risk of false-negative cases being missed. As in the original reports noted above, difficulties of discrimination in static images derived from small cells which may be difficult to classify and cells that are out of focus [26].

In nongynecologic applications, a variety of organ system telecytology studies have been reported. Galvez et al. [27], reporting on the specific cytologic features identified in breast fine-needle aspirations, noted that all features assessed could be identified in both the static images and under the microscope. They reported that discordant cases were almost always the difference between reporting a 'suspicious' versus a 'definitive' interpretation of malignancy. In another breast fine-needle aspiration study of 48 cases, Della Mea et al. [28] reported that using static images sent by e-mail achieved a satisfactory result in $84 \%$ of cases. In the remaining $16 \%$, the images were deemed insufficient for a definitive diagnosis, with 1 case representing a false-negative result in an invasive lobular carcinoma. Briscoe et al. [29] reported that observer experience was important in breast fine-needle aspiration static-image telecytology. In their study, the pathologist having the most experience had the highest level of concordance and 'diagnostic confidence'. Ayatollahi et al. [30] reported between 83 and $87 \%$ accuracy for the interpretation of static images from pleural effusion specimens compared to the final diagnosis versus $89 \%$ accuracy for interpretation of the glass slides. In studies of pancreatic fine-needle aspiration biopsies by static images, Marchevsky et al. [31] reported improved performance over light-microscopic examination in the discrimination of chronic pancreatitis from low-grade adenocarcinoma. They did report considerable inter- and intraobserver variability in diagnosis, which might be expected for this often difficult discrimination, but less was reported using the static-image approach. This conclusion might be predicted on the fact that the images selected might be the best representations of the pathology present, and would not be 'diluted' by all the other appearances on the entire glass slide. This hypothesis speaks to the fact that image selection quality and bias may play an important role in the variable quality of static-image telecytology. For a variety of aspiration specimens, Jialdasani et al. [32] reported a 'clinically useful' diagnosis in 91\% of cases using static-image telecytology. Yamashiro et al. [33] report on their introduction of a static imagebased telecytology system for primary diagnosis in Japan. Over a 2-year period, 614 routine cases were transmitted by cytotechnologists to pathologists working $300 \mathrm{~km}$ away, with case discussion simultaneously via e-mail. The concordance between telecytology and subsequent glass slide interpretation was $88.6 \%$. The overall accuracy was $91.4 \%$, and was not statistically different from the 'glass slide by mail' accuracy noted in the prior year. There were only 5 cases $(0.8 \%)$ in which a 'severely inappropriate diagnosis' was made by telecytology. These cases were all difficult differential diagnoses, including lymphoma, bile duct and salivary gland neoplasia, in which routine glass slide diagnosis might be fraught with difficulty. The authors conclude that this system worked adequately for routine use [33]. Other examples of staticimage cytology have shown excellent accuracy and reproducibility among observers in a variety of organ systems and even for use in veterinary cytology practice [34-37].

Interestingly, other factors may have additional influences on the diagnostic accuracy of static-image telecytology, most notably manipulations of the images themselves in various ways. In a timely study, Pinco et al. [38] presented images to observers at multiple times with changes in the physical characteristics of the image files - including changes in presentation (rotation), brightness, contrast, red-green-blue color, and luminosity (all manipulated via a Photoshop application). The authors noted that agreement between identical images was excellent $(\kappa=0.81)$, but was reported to be poor $(\kappa=0.21)$ when the images were manipulated, and these differences were statistically significant. Examples of issues include changes occurring via cropping of images that may highlight different cells or orientations, changes in density of nuclei (making cells appear more hyperchromatic), or changes in the background color causing obscuration of important foreground details. This study argues strongly for community standards of these factors going forward, but also for increased awareness that manipulating images may lead to erroneous results.

There have been few examples of real-time telecytology scanning studies for primary or secondary consultation that could be identified in the literature. This is not surprising given the issues noted above. This method of examination is more amenable to low-magnification work, with only the occasional need for increased magnification to verify the low-power impression. Hence this technique has been used almost exclusively in cytology for rapid assessment - where final diagnoses will be made later on the collected material. Tasks such as high-magnification searching and screening are not well suited to this technique because of the time and bandwidth needed. These dynamic systems are more adaptable to educa- 
tion environments where lower-resolution real-time images can be used to teach. In one study using real-time assessment for breast fine-needle aspiration cytology evaluation, Hitchcock and Hitchcock [39] reported a significant discordant rate (greater than 1 step) of 3.8\%, with 5 cases upgraded and 3 cases downgraded on final diagnosis. They report that these results are acceptable and they continued to use this system in current practice. Another report using robotic real-time methods for breast telecytologic interpretation showed correlation in $80 \%$ of cases, and also documented a substantial increase in time taken to review the telecytology specimen (10.8 vs. 2.4 min per specimen). They concluded that the method was not ready for routine use, but indicated that the results were 'encouraging' [9].

The use of whole-slide imaging in clinical cytology specimens has recently been reported. As mentioned above, whole-slide images are complete digital representations of the entire cytology specimen. As such, the issues of bias and incomplete specimens for remote evaluation are eliminated. However, whole-slide images, as they are for all intents and purposes very large static images, still suffer from lack of intrinsic focusing capability and are limited in resolution by the technical features of their acquisition (i.e. what is the original capture optical magnification). z-Stacking and integration of multiplanar scans can mitigate focusing issues but come with the inherent costs of long scanning and/or transmission times. A variety of devices are currently available for the making of whole-slide images. A review of current devices was reported in 2006 [40], and serves as a starting point for interested readers; however, the field is in a constant and rapid state of change, and hence the newest information on current scanning devices is generally only available from the manufacturers themselves. Many studies of whole-slide imaging technology are available for histopathologic applications, including frozen section and permanent slide applications [2, 41-50]. Only a few reports detail the use of whole-slide images in cytology diagnostic applications $[3,51,52]$. In a recent study by Evered and Dudding [3], using whole-slide images for the evaluation of liquid-based cervical cytology slides, there was no statistical difference in accuracy between wholeslide images and glass slides; however, there were important differences noted. Time to screen the whole-slide images more than doubled compared to the glass slide (18 vs. $8 \mathrm{~min}$, respectively), and it was noted that whole-slide images underperformed glass slides when images were captured in single planes, without focusing capability, as compared to multiplane z-stacks, where focusing was en- abled. They also reported significant computer issues, including 'freezing' of the program, 'fuzzy' images and slow computer response times. In a study of the use of virtual slides to prescreen and evaluate cervical cytology slides which were stained with the p16 biomarker for the detection of potential high-grade disease, Grabe et al. [52] showed that the device's ability to detect stained cells on whole-slide images had a very high sensitivity and specificity when compared to the gold standard. This study shows that whole-slide images are well suited to further artificial-intelligence functions. It is anticipated that cytologic evaluations by whole-slide imaging will dramatically increase in the next few years, as technical issues as described are addressed.

For interested readers, an excellent review of the data available for telecytology clinical studies using a variety of systems has been published by Pantanowitz et al. [53].

\section{Digital Cytology Applications in Education}

A great advantage of the use of digital cytology is its ease of use in teaching applications. In an age of ever more expensive and difficult travel, the need to bring the education to the student increases. Because of their ability to access any site with a fast Internet connection, digital cytology education methods have been used in a variety of circumstances. The use of static images in a presentation program transmitted over the Internet can accompany a voice connection for distance lecturing. Use of real-time Internet presentations where students can view the sender's computer screen (so-called 'webinars') can be useful not only for lectures but also for microscopy teaching, when the computer is coupled to a microscope with camera. In this fashion, other microscopy-related conferencing, such as tumor boards or morbidity and mortality conferences, can be easily performed from a distance [54]. In specific telecytology applications, educational tools have been used to teach cytotechnologist locator skills using virtual slides. In this application, students screened validated cytology whole-slide images to which their annotations could be compared to reference annotations, and then wiped clean again for the next session [55]. With the buildup of sets of static or wholeslide images in such a fashion, excellent examples of rare cases could be archived for widespread use without issues of stain degradation, slide breakage or loss, and such images could be stored in a structured database which would allow for rapid search and retrieval. Digital cytology has also been successfully evaluated for use in 
educational challenges and examinations [56, 57]. Many of the pathology and cytology professional organizations now offer digital online educational experiences, both free and by subscription. Interested viewers can access many of these exercises at the websites of the organizations (e.g. www.cytology-iac.org, www.cytopathology. org, www.cap.org, www.ascp.org, www.uscap.org, www. eurocytology.eu).

\section{Use of Digital Cytology for Proficiency Testing}

At present, gynecologic cytology proficiency testing is mandatory for all United States cytologists practicing in this field. This program is currently mandated by law to be administered using glass slide-based test sets. In order to test all cy tologists every year, testing providers need to obtain, validate and maintain large sets of glass slides. In addition, testing sets need to be physically transported to each laboratory. This situation argues strongly for a 'digital solution.' Libraries of whole-slide images would be far more easily maintained and would not be subject to loss in transport and the inevitable wear and tear of repeated use. Early studies by the Centers for Disease Control and Prevention showed that either portions of slides or whole-slide images could be used for this task as long as the slides were well validated $[58,59]$. When wholeslide images were compared to glass slides, there was no statistical difference for the modeled proficiency test pass rate [59]. In other studies, Marchevsky et al. [60] and Stewart et al. [61] showed that optimal performance on whole-slide image-based proficiency testing requires user training and experience, and may be hindered by 'interface' issues such as the ability to move quickly and easily around the virtual slide, to change magnifications and to focus.

Based on the rapid evolution of technology in wholeslide imaging, the 'engineering' issues of image capture and viewing will undoubtedly improve. With these changes, as well as with increasing levels of experience and skill in the cytology community, proficiency testing should be an ideal 'target' for the use of this technology.

\section{Administrative, Legal, and Regulatory Issues in Digital Cytology}

With implementation of applications of digital cytology (and general digital pathology) into actual practice and patient care settings, issues related to billing, secu- rity, and the medicolegal and regulatory aspects of practice will need to be addressed.

At present, billing for teleconsulations is identical to billing for glass slide consultations. This could change in the future as increased 'traffic' may invoke further review and initiation of new or more specific codes and fee structures.

In the era of telemedicine, confidentiality of protected patient information and verification of correct transmission are critical. The sending of information 'in the clear' over the Internet is not secure and hence additional measures must ensue for transmission of protected information during the process of teleconsultation. In point-topoint teleconsultation (meaning always between the same places), use of a virtual private network may be an optimal choice as a secure link. However, in an 'open' referral system where consultations can come from a variety of sites, this is not a good option. In such cases, images (which are not - at least at present - considered protected information) could be transmitted over the Internet, but the accompanying patient information would have to come separately (telephone or fax) or by another encrypted method, such as secure sockets layer (SSL), if attached to the image information - similar to the method for credit card security used in e-commerce [7].

In the era of so-called 'cloud' computing, where information is stored in a commercial server outside of either the sender's or receiver's firewalls, these security issues become even more important and have yet to be completely worked out.

Teleconsultation licensure requirements vary by state and therefore knowledge of specific state law is necessary. Most states require licensure for primary interpretation. Some states have virtually no restrictions on secondary review of cases while others have restrictions based on billing patterns or case volumes.

Medicolegal issues associated with digital practice will likely be similar to those related to standard glass slide microscopy practice. There will undoubtedly be nuances associated with teleconsultation, however. For instance, in real-time teleconsultation, is the consultant responsible if the sender does not show the most appropriate fields of view; how would the review be documented? In another example, if the quality of a whole-slide image does not show many of the relevant cells with the captured focal plane, who bears the responsibility? These and many other issues will arise and will require cases and courts to be satisfactorily defined and resolved $[62,63]$. 
The College of American Pathologists (CAP) Laboratory Accreditation Program (LAP) checklists do have items relevant for digital pathology and teleconsultation. They include: (1) the necessity of having systems in place to insure that patient identification and the images associated are correctly linked; (2) all systems are compliant with the Health Insurance Portability and Accountability Act (HIPAA; patient confidentiality); (3) a system is in place to insure that clinical information is available to the consultant (just as for glass slide reviews, teleconsulations require clinical history); (4) adequate training and documentation thereof is required for users of teleconsultation equipment - from scanning to transmission to viewing to reporting; (5) quality assurance procedures are in place (not stated as to what these might be - user defined), and (6) that systems used are adequate for their stated use (for instance, use of scanners of appropriate resolution for cytology might be an example). For further information, the interested reader should review the CAP checklists which can be found on the CAP website (www.cap.org).

It is likely that there will be significant change in the regulatory environment for digital pathology in the next decade. The United States Food and Drug Administration (FDA) recently held a conference to begin investigations into the regulation of this field. Issues of instrument and process validation, patient safety and confidentiality, and regulation of telepathology are certain to be addressed by the FDA in the near future.

Lastly, in order to set up a teleconsultation practice, information technology (IT) departments in institutions must be engaged. IT department personnel must insure that bandwidth is adequate for the expected information load and that the proper linkages (ports) for each station are adequate for the anticipated data flow. IT personnel must insure that systems are compliant with HIPAA regulations and that security of information is adequate. IT must insure that the institutional firewall is adequate to prevent unwanted access to information and that teleconsultation material is capable of getting through and reports are capable of getting back out again. IT will need to insure that host servers have adequate virus protection and security measures in place and that there is a mechanism to scan incoming material for security threats. Finally, IT needs to help in setting up compatible systems with other institutions. This will require them to interact with pathology departments and with IT units at other institutions. In most large centers, IT has been tasked with numerous complex tasks related to laboratory and clinical information systems. Having administrative support specifically for teleconsultation services is critical to insure that IT personnel are engaged in this process. Such engagement may require dedicated teleconsultation personnel, particularly if a large ongoing volume is anticipated.

\section{Prospects for the Future of Digital Cytology}

At the current developmental pace, it is likely that most of the issues noted above will be worked out satisfactorily in the next several years and that digital sharing of cases within or from outside of departments will be commonplace. In the future, the addition of artificial intelligence and data mining will likely be the next level of digital pathology technology. Already in gynecologic cytology, devices are capable of identifying and ranking potentially abnormal cells, with presentation of these areas digitally to observers for assessments [41]. Transfer of this capability to whole-slide digital images would allow for flexibility in the process because analysis of the slides would not be tied to the physical presence of the slides. In addition, manipulation of digital information and relationships might provide additional diagnostic information. Work has already begun on similar processes in histopathology applications [64]. Perhaps in the future pathologists will look at prescreened digital slides which have regions of interest highlighted by computer scanners trained to effectively identify high-risk patterns. Primary applications might be in areas requiring intensive screening of large numbers of sections (e.g. prostate or breast biopsies) or in nongynecologic cytology screening applications.

The future of digital pathology is bright and should usher in entirely new ways for pathologists to interact with specimens. These methods are already widely available for educationsal use. Use for patient teleconsultation, both within institutions and between institutions, promises to improve the efficiency of practice and also to improve patient safety. High-quality primary interpretations and secondary opinions on routine and difficult cases will be only minutes away from any site in the world.

It is certain that the new generation of cytologists and pathologists being trained today will more easily adapt to this technology. In the era of virtually unlimited access to 'on-line' information, and use of rapidly improving interfaces to access this information, the practitioners of tomorrow will not only embrace this method of practice, but will likely demand it. 


\section{References}

1 Yamashiro K, Taira K, Matsubayashi S, Azuma M, Okuyama D, Nakajima M, Takeda $H$, Suzuki H, Kawamura N, Wakao F, Yagi Y: Comparison between a traditional single still image and a multiframe video image along the $\mathrm{z}$-axis of the same microscopic field of interest in cytology: which does contribute to telecytology? Diagn Cytopathol 2009;37:727-731.

-2 Weinstein RS, Descour MR, Liang C, Barker G, Scott KM, Richter L, Krupinski EA, Bhattacharyya AK, Davis JR, Graham AR, Rennels M, Russum WC, Goodall JF, Zhou P, Olszak AG, Williams BH, Wyant JC, Bartels $\mathrm{PH}$ : An array microscope for ultrarapid virtual slide processing and telepathology. Design, fabrication, and validation study. Hum Pathol 2004;35:1303-1414.

3 Evered A, Dudding N: Accuracy and perceptions of virtual microscopy compared with glass slide microscopy in cervical cytology. Cytopathology 2010 May 17. DOI: 10.1111/ j.1365-2303.2010.00758.x.

$\checkmark 4$ Dee FR, Donnelly A, Radio S, Leaven T, Zaleski MS, Kreiter C: Utility of 2-D and 3-D virtual microscopy in cervical cytology education and testing. Acta Cytol 2007;51:523529.

$>5$ Weinstein RS, Descour MR, Liang C, Bhattacharyya AK, Graham AR, Davis JR, Scott KM, Richter L, Krupinski EA, Szymus J, Kayser K, Dunn BE: Telepathology overview: from concept to implementation. Hum Pathol 2001;32:1283-1299.

$\checkmark 6$ Weinstein RS, Graham AR, Richter LC, Barker GP, Krupinski EA, Lopez AM, Erps KA, Bhattacharyya AK, Yagi Y, Gilbertson JR: Overview of telepathology, virtual microscopy, and whole slide imaging: prospects for the future. Hum Pathol 2009;40:10571069.

7 Williams S, Henricks WH, Becich MJ, Toscano M, Carter AB: Telepathology for patient care: what am I getting myself into? Adv Anat Pathol 2010;17:130-149.

$>8$ O’Brien MJ, Takahashi M, Brugal G, Christen H, Gahm T, Goodell RM, Karakitsos P, Knesel EA Jr, Kobler T, Kyrkou KA, Labbe S, Long EL, Mango LJ, McGoogan E, Oberholzer M, Reith A, Winkler C: Digital imagery/ telecytology. International Academy of Cytology Task Force summary. Diagnostic Cytology Towards the 21st Century: An International Expert Conference and Tutorial. Acta Cytol 1998;42:148-164.

>9 Singh N, Akbar N, Sowter C, Lea KG, Wells $\mathrm{CA}$ : Telepathology in a routine clinical environment: implementation and accuracy of diagnosis by robotic microscopy in a onestop breast clinic. J Pathol 2002;196:351-355.

$>10$ Kim B, Chhieng D, Jhala N, et al: Dynamic telecytopathology has equivalent efficacy with on site rapid cytology diagnosis for pancreatic carcinoma. Cancer Cytopathol 2006; 108:357.
11 Delta Mea V, Cataldi P, Pertoldi B, Beltrami CA: Dynamic robotic telepathology: a preliminary evaluation on frozen sections, histology and cytology. J Telemed Telecare 1999;5(suppl 1):S55-S56.

12 Kerr SE, Bellizzi AM, Stelow EB, Frierson HF Jr, Policarpio-Nicolas ML: Initial assessment of fine-needle aspiration specimens by telepathology: validation for use in pathology resident-faculty consultations. Am J Clin Pathol 2008;130:409-413.

13 Alsharif M, Carlo-Demovich J, Massey C, Madory JE, Lewin D, Medina AM, Recavarren R, Houser PM, Yang J: Telecytopathology for immediate evaluation of fine-needle aspiration specimens. Cancer Cytopathol 2010;118:119-126.

14 Heimann A, Maini G, Hwang S, Shroyer KR, Singh M: Use of telecytology for the immediate assessment of CT guided and endoscopic FNA cytology: diagnostic accuracy, advantages, and pitfalls. Diagn Cytopathol 2010 Dec 3 [Epub ahead of print].

15 Kaplan KJ: Telecytopathology for immediate evaluation of fine-needle aspiration specimens. Cancer Cytopathol 2010;118:115-118.

16 Weinstein RS: Telepathology comes of age in Norway. Hum Pathol 1991;22:511-513.

17 Raab SS, Zaleski MS, Thomas PA, Niemann $\mathrm{TH}$, Isacson C, Jensen CS: Telecytology: diagnostic accuracy in cervical-vaginal smears. Am J Clin Pathol 1996;105:599-603.

18 Ziol M, Vacher-Lavenu MC, Heudes D, Ferrand J, Mayelo V, Molinie V, Slama S, Marsan C: Expert consultation for cervical carcinoma smears. Reliability of selected-field videomicroscopy. Anal Quant Cytol Histol 1999;21:35-41.

19 Alli PM, Ollayos CW, Thompson LD, Kapadia I, Butler DR, Williams BH, Rosenthal DL, O’Leary TJ: Telecytology: intraobserver and interobserver reproducibility in the diagnosis of cervical-vaginal smears. Hum Pathol 2001;32:1318-1322.

20 Allen EA, Ollayos CW, Tellado MV, Butler DR, Buckner SB, Williams BH, O'Leary TJ: Characteristics of a telecytology consultation service. Hum Pathol 2001;32:13231326.

21 Lee ES, Kim IS, Choi JS, Yeom BW, Kim HK, Han JH, Lee MS, Leong AS: Accuracy and reproducibility of telecytology diagnosis of cervical smears. A tool for quality assurance programs. Am J Clin Pathol 2003;119:356360 .

22 Wilbur DC, Prey MU, Miller WM, Pawlick GF, Colgan TJ: The AutoPap system for primary screening in cervical cytology. Comparing the results of a prospective, intendeduse study with routine manual practice. Acta Cytol 1998;42:214-220.
23 Wilbur DC, Madi K, Colvin RB, Duncan LM, Faquin WC, Ferry JA, Frosch MP, Houser SL, Kradin RL, Lauwers GY, Louis DN, Mark EJ, Mino-Kenudson M, Misdraji J, Nielsen GP, Pitman MB, Rosenberg AE, Smith RN, Sohani AR, Stone JR, Tambouret $\mathrm{RH}, \mathrm{Wu} \mathrm{CL}$, Young RH, Zembowicz A, Klietmann W: Whole-slide imaging digital pathology as a platform for teleconsultation: a pilot study using paired subspecialist correlations. Arch Pathol Lab Med 2009;133: 1949-1953.

24 Dziura B, Quinn S, Richard K: Performance of an imaging system vs. manual screening in the detection of squamous intraepithelial lesions of the uterine cervix. Acta Cytol 2006;50:309-311.

25 Eichhorn JH, Brauns TA, Gelfand JA, Crothers BA, Wilbur DC: A novel automated screening and interpretation process for cervical cytology using the internet transmission of low-resolution images: a feasibility study. Cancer 2005;105:199-206.

26 Eichhorn JH, Buckner L, Buckner SB, Beech DP, Harris KA, McClure DJ, Crothers BA, Wilbur DC: Internet-based gynecologic telecytology with remote automated image selection: results of a first-phase developmental trial. Am J Clin Pathol 2008;129:686-696.

27 Galvez J, Howell L, Costa MJ, Davis R: Diagnostic concordance of telecytology and conventional cytology for evaluating breast aspirates. Acta Cytol 1998;42:663-667.

28 Della Mea V, Puglisi F, Bonzanini M, Forti S, Amoroso V, Visentin R, Dalla Palma P, BeltramiCA: Fine-needle aspiration cytology of the breast: a preliminary report on telepathology through Internet multimedia electronic mail. Mod Pathol 1997;10:636-641.

29 Briscoe D, Adair CF, Thompson LD, Tellado MV, Buckner SB, Rosenthal DL, O'Leary TJ: Telecytologic diagnosis of breast fine needle aspiration biopsies. Intraobserver concordance. Acta Cytol 2000;44:175-180.

30 Ayatollahi H, Khoei A, Mohammadian N, Sadeghian MH, Azari JB, Ghaemi MR, Khoob MK: Telemedicine in diagnostic pleural cytology: a feasibility study between universities in Iran and the USA. J Telemed Telecare 2007;13:363-368.

31 Marchevsky AM, Nelson V, Martin SE, Greaves TS, Raza AS, Zeineh J, Cobb CJ: Telecytology of fine-needle aspiration biopsies of the pancreas: a study of well-differentiated adenocarcinoma and chronic pancreatitis with atypical epithelial repair changes. Diagn Cytopathol 2003;28:147-152.

32 Jialdasani R, Desai S, Gupta M, Kothari A, Deshpande R, Shet T, Ramadwar M, Kane S, Chinoy R: An analysis of 46 static telecytology cases over a period of two years. J Telemed Telecare 2006;12:311-314. 
-33 Yamashiro K, Kawamura N, Matsubayashi S, Dota K, Suzuki H, Mizushima H, Wakao F, Azumi N: Telecytology in Hokkaido Island, Japan: results of primary telecytodiagnosis of routine cases. Cytopathology 2004; 15: 221-227.

34 Georgoulakis J, Archondakis S, Panayiotides I, Anninos D, Skagias L, Stamataki M, Peros G, Karakitsos P: Study on the reproducibility of thyroid lesions telecytology diagnoses based upon digitized images. Diagn Cytopathol 2010 Aug 20 [Epub ahead of print].

- 35 Archondakis S, Georgoulakis J, Stamataki M, Anninos D, Skagias L, Panayiotides I, Peros G, Karakitsos P: Telecytology: a tool for quality assessment and improvement in the evaluation of thyroid fine-needle aspiration specimens. Telemed J E Health 2009;15:713717.

-36 Maiolino P, Restucci B, Papparella S, De Vico G: Evaluation of static telepathology in veterinary diagnostic cytology. Vet Clin Pathol 2006;35:303-306.

- 37 Kayser K, Kayser G, Becker HD, Herth F: Telediagnosis of transbronchial fine needle aspirations - a feasibility study. Anal Cell Pathol 2000;21:207-212.

- 38 Pinco J, Goulart RA, Otis CN, Garb J, Pantanowitz L: Impact of digital image manipulation in cytology. Arch Pathol Lab Med 2009;133:57-61.

-39 Hitchcock CL Hitchcock LE: Three years of experience with routine use of telepathology in assessment of excisional and aspirate biopsies of breast lesions. Croat Med J 2005;46: 449-457.

-40 Rojo MG, Garcia GB, Mateos CP, Garcia JG, Vicente MC: Critical comparison of $31 \mathrm{com}$ mercially available digital slide systems in pathology. Int J Surg Pathol 2006;14:285305.

41 Wilbur DC, Black-Schaffer WS, Luff RD, Abraham KP, Kemper C, Molina JT, Tench WD: The Becton Dickinson FocalPoint GS Imaging System: clinical trials demonstrate significantly improved sensitivity for the detection of important cervical lesions. Am J Clin Pathol 2009;132:767-775.

42 Fallon MA, Wilbur DC, Prasad M: Ovarian frozen section diagnosis: use of whole-slide imaging shows excellent correlation between virtual slide and original interpretations in a large series of cases. Arch Pathol Lab Med;134:1020-1023.
43 Li X, Liu J, Xu H, Gong E, McNutt MA, Li F, Anderson VM, Gu J: A feasibility study of virtual slides in surgical pathology in China. Hum Pathol 2007;38:1842-1848.

44 Furness P: A randomized controlled trial of the diagnostic accuracy of internet-based telepathology compared with conventional microscopy. Histopathology 2007;50:266273.

45 Rojo MG, Gallardo AJ, Gonzalez L, Peces C, Murillo C, Gonzalez J, Sacristan J: Reading virtual slide using web viewers: results of subjective experience with three different solutions. Diagn Pathol 2008;3(suppl 1):S23.

46 Fine JL, Grzybicki DM, Silowash R, Ho J, Gilbertson JR, Anthony L, Wilson R, Parwani AV, Bastacky SI, Epstein JI, Jukic DM: Evaluation of whole slide image immunohistochemistry interpretation in challenging prostate needle biopsies. Hum Pathol 2008; 39:564-572.

47 Slodkowska J, Chyczewski L, Wojciechowski M: Virtual slides: application in pulmonary pathology consultations. Folia Histochem Cytobiol 2008;46:121-124.

48 Ho J, Parwani AV, Jukic DM, Yagi Y, Anthony L, Gilbertson JR: Use of whole slide imaging in surgical pathology quality assurance: design and pilot validation studies. Hum Pathol 2006;37:322-331.

49 Gilbertson JR, Ho J, Anthony L, Jukic DM, Yagi Y, Parwani AV: Primary histologic diagnosis using automated whole slide imaging: a validation study. BMC Clin Pathol 2006;6: 4

-50 Molnar B, Berczi L, Diczhazy C, Tagscherer A, Varga SV, Szende B, Tulassay Z: Digital slide and virtual microscopy based routine and telepathology evaluation of routine gastrointestinal biopsy specimens. J Clin Pathol 2003;56:433-438.

51 Demichelis F, Barbareschi M, Dalla Palma P, Forti S: The virtual case: a new method to completely digitize cytological and histological slides. Virchows Arch 2002;441:159164.

52 Grabe N, Lahrmann B, Pommerencke T, von Knebel Doeberitz M, Reuschenbach M, Wentzensen N: A virtual microscopy system to scan, evaluate and archive biomarker enhanced cervical cytology slides. Cell Oncol 2010;32:109-119.

53 Pantanowitz L, Hornish M, Goulart RA: The impact of digital imaging in the field of cytopathology. Cytojournal 2009;6:6.
54 Mulford DK: Telepathology education: Reaching out to cytopathology programs throughout the country. ASC Bull 2006;43: 25-30.

55 Stewart J, 3rd, Bevans-Wilkins K, Bhattacharya $\mathrm{A}$, Ye C, Miyazaki K, Kurtycz DF: Virtual microscopy: an educator's tool for the enhancement of cytotechnology students' locator skills. Diagn Cytopathol 2008;36: 363-368.

56 Neel JA, Grindem CB, Bristol DG: Introduction and evaluation of virtual microscopy in teaching veterinary cytopathology. J Vet Med Educ 2007;34:437-444.

57 Glatz K, Willi N, Glatz D, Barascud A, Grilli B, Herzog M, Dalquen P, Feichter G, Gasser TC, Sulser T, Bubendorf L: An international telecytologic quiz on urinary cytology reveals educational deficits and absence of a commonly used classification system. Am J Clin Pathol 2006;126:294-301.

58 Taylor RN, Gagnon M, Lange J, Lee T, Draut R, Kujawski E: CytoView. A prototype computer image-based Papanicolaou smear proficiency test. Acta Cytol 1999;43:1045-1051.

-59 Gagnon M, Inhorn S, Hancock J, Keller B, Carpenter D, Merlin T, Hearn T, Thompson P, Whalen R: Comparison of cytology proficiency testing: glass slides vs. virtual slides. Acta Cytol 2004;48:788-794.

60 Marchevsky AM, Khurana R, Thomas P, Scharre K, Farias P, Bose S: The use of virtual microscopy for proficiency testing in gynecologic cytopathology: a feasibility study using ScanScope. Arch Pathol Lab Med 2006;130:349-355.

61 Stewart J 3rd, Miyazaki K, Bevans-Wilkins K, Ye C, Kurtycz DF, Selvaggi SM: Virtual microscopy for cytology proficiency testing: are we there yet? Cancer 2007;111:203-209.

62 Tsuchihashi Y, Okada Y, Ogushi Y, Mazaki T, Tsutsumi Y, Sawai T: The current status of medicolegal issues surrounding telepathology and telecytology in Japan. J Telemed Telecare 2000;6(suppl 1):S143-S145.

63 Leung ST, Kaplan KJ: Medicolegal aspects of telepathology. Hum Pathol 2009;40:11371142.

64 Kayser K, Radziszowski D, Bzdyl P, Sommer R, Kayser G: Towards an automated virtual slide screening: theoretical considerations and practical experiences of automated tissue-based virtual diagnosis to be implemented in the Internet. Diagn Pathol 2006; 10. 